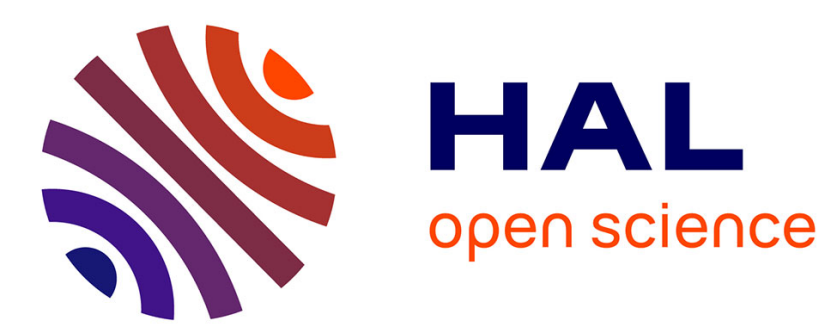

\title{
Optimal velocity and density profiles for the onset of absolute instability in jets
}

Lutz Lesshafft, Olivier Marquet

\section{To cite this version:}

Lutz Lesshafft, Olivier Marquet. Optimal velocity and density profiles for the onset of absolute instability in jets. Journal of Fluid Mechanics, 2010, 662 (November), pp.398-408. 10.1017/s0022112010004246 . hal-01020674

\section{HAL Id: hal-01020674 \\ https://hal-polytechnique.archives-ouvertes.fr/hal-01020674}

Submitted on 9 Jul 2014

HAL is a multi-disciplinary open access archive for the deposit and dissemination of scientific research documents, whether they are published or not. The documents may come from teaching and research institutions in France or abroad, or from public or private research centers.
L'archive ouverte pluridisciplinaire HAL, est destinée au dépôt et à la diffusion de documents scientifiques de niveau recherche, publiés ou non, émanant des établissements d'enseignement et de recherche français ou étrangers, des laboratoires publics ou privés. 


\title{
Optimal velocity and density profiles for the onset of absolute instability in jets
}

\author{
LUTZ LESSHAFFT ${ }^{1} \dagger$ AND OLIVIER MARQUET ${ }^{2}$ \\ ${ }^{1}$ Laboratoire d'Hydrodynamique, CNRS - École Polytechnique, 91128 Palaiseau, France \\ ${ }^{2}$ ONERA DAFE, 8 rue des Vertugadins, 92190 Meudon, France
}

(Received 26 February 2010; revised 31 July 2010; accepted 9 August 2010; first published online 27 September 2010)

\begin{abstract}
The absolute/convective character of the linear instability of axisymmetric jets is investigated for a wide range of parallel velocity and density profiles. An adjointbased sensitivity analysis is carried out in order to maximize the absolute growth rate of jet profiles with and without density variations. It is demonstrated that jets without counterflow may display absolute instability at density ratios well above the previously assumed threshold $\rho_{\text {jet }} / \rho_{\infty}=0.72$, and even in homogeneous settings. Absolute instability is promoted by a strong velocity gradient in the low-velocity region of the shear layer, as well as by a step-like density variation near the location of maximum shear. A new efficient algorithm for the computation of the absolute instability mode is presented.
\end{abstract}

Key words: absolute/convective instability, instability, instability control

\section{Introduction}

The spontaneous onset of self-excited oscillations in low-density axisymmetric jets has been linked to the presence of absolute instability in the unperturbed underlying baseflow since their first experimental observation by Monkewitz et al. (1990). Numerous experimental and numerical studies have confirmed this hypothesis (e.g. Hallberg et al. 2007; Lesshafft, Huerre \& Sagaut 2007, and references therein). One important implication of this association is that linear stability theory may predict the range of parameters, and in particular the ratio of jet density over ambient density, over which self-excited oscillations may occur in real jets.

Monkewitz \& Sohn (1988) were the first to investigate the absolute/convective instability properties of low-density jets. For a specific family of analytical velocity and density profiles, designed to match experimental measurements, a transition from convective to absolute instability was found to be possible when the density ratio $S=\rho_{j e t} / \rho_{\infty}$ fell below the critical value $S_{\text {crit }}=0.72$. This value was obtained for the inviscid, zero-Mach-number case, and for a velocity profile with a thin shear layer of momentum thickness $\theta_{u}=0.042$, non-dimensionalized with the jet radius, as it may be found in the potential core region close to the nozzle exit. Lower Reynolds and higher Mach numbers, as well as thicker shear layers, were shown to require stronger density differences in order to admit absolute instability. Furthermore, for baseflow profiles typical of the potential core, absolute instability was always found to occur first for axisymmetric perturbations. Later linear instability studies by Jendoubi \& 
Strykowski (1994) and Lesshafft \& Huerre (2007) corroborated the validity of all the above observations for a different family of analytical profiles, introduced by Michalke (1971). The critical density ratio for this profile family was determined to be $S_{\text {crit }}=0.713$, for a momentum thickness of $\theta_{u}=0.039$. It has since been generally assumed that these critical values are approximately valid regardless of the precise functional shape of the velocity profile, which would then be sufficiently characterized by its momentum thickness. The density profile in all these studies follows directly from the velocity profile via the Crocco-Busemann relation.

Coenen, Sevilla \& Sánchez (2008) integrated the boundary layer equations in order to obtain velocity profiles of low-density jets issuing from a straight pipe. Their computations account for the growth of a laminar boundary layer inside the pipe; at the nozzle exit, the density profile is prescribed as a step function. The analysis of Coenen et al. (2008) showed that absolute instability in such configurations may set in at a density ratio $S=0.9$, for velocity profiles with a fairly thick shear layer $\theta_{u}=0.1$. This remarkable result clearly demonstrates that the restriction to the previously studied analytical velocity profile shapes, and to density profiles governed by the Crocco-Busemann relation, is inadequate for determining a general upper bound for the density ratio of absolutely unstable jets.

The principal objective of the present study is to assess whether such an upper bound of $S$ for the onset of absolute instability can be established. Towards this end, many limiting assumptions about the shape of velocity and density profiles will be successively relaxed. A sensitivity-based optimization procedure is employed to identify profile shapes that display absolute instability at a minimal density difference. The methodology is similar to that of Bottaro, Corbett \& Luchini (2003) and Hwang \& Choi (2006). All previous jet instability studies, including that of Coenen et al. (2008), indicate that absolute instability can be expected to first arise for axisymmetric perturbations, at infinite Reynolds number and zero Mach number. The present investigation is therefore limited to those settings. The effects of jet swirl and of counterflow in the outer stream, both of which may also give rise to absolute instability (Jendoubi \& Strykowski 1994; Gallaire \& Chomaz 2003), are excluded from the analysis.

The governing equations and numerical methods are presented in $\S 2$. In particular, the adjoint-based sensitivity analysis is laid out, and a novel efficient algorithm for calculating the absolute instability mode is introduced. Section 3 offers a parametric study of an analytical profile family, where velocity and density are no longer linked by the Crocco-Busemann law. In $\S 4$, the restriction to analytical profile shapes is dropped. Free optimization yields baseflow profiles that require a minimal density difference in order to be absolutely unstable. An argument for the early onset of absolute instability is derived from the shape of the eigenmodes.

\section{Numerical model}

\subsection{Direct and adjoint temporal dispersion relations}

The linear stability analysis in the present study is restricted to axisymmetric disturbances in inviscid jets at zero Mach number. Perturbations of the streamwise velocity $u^{\prime}$, radial velocity $v^{\prime}$ and pressure $p^{\prime}$ are assumed to take the form of normal modes $\left[u^{\prime}, v^{\prime}, p^{\prime}\right](x, r, t)=[U(r), V(r), P(r)] \exp (\mathrm{i} \omega t-\mathrm{i} k x)$, where $x$ and $r$ are the streamwise and radial coordinates, $\omega$ is the complex circular frequency and $k$ is the complex wavenumber. A parallel baseflow is defined by the velocity and density profiles $u_{b}(r)$ and $\rho_{b}(r)$. All quantities are made non-dimensional with respect to the jet 
radius and the velocity and density values on the jet axis at $r=0$. In the inviscid, zeroMach-number limit, the linear perturbation equations given in Lesshafft \& Huerre (2007) reduce to

$$
\begin{aligned}
k u_{b} U-\mathrm{i} V \partial_{r} u_{b}+k \rho_{b}^{-1} P & =\omega U, \\
k u_{b} V-\mathrm{i} \rho_{b}^{-1} \partial_{r} P & =\omega V, \\
\partial_{r} V+r^{-1} V+\mathrm{i} k U & =0,
\end{aligned}
$$

with boundary conditions $\partial_{r} U=V=\partial_{r} P=0$ on the jet axis. Note that density perturbations at zero Mach number are decoupled from the velocity and pressure perturbations, and do not need to be solved for. Equations (2.1)-(2.3) are discretized using Chebyshev collocation, with 200 collocation points distributed over the interval $0 \leqslant r \leqslant r_{\max }=100$, according to the same mapping function as used in Lesshafft \& Huerre (2007). The equations are then cast in the form of the discrete temporal eigenvalue problem $\boldsymbol{A} \boldsymbol{q}=\omega \boldsymbol{B} \boldsymbol{q}$, where the eigenvector $\boldsymbol{q}=(\boldsymbol{U}, \boldsymbol{V}, \boldsymbol{P})^{\mathrm{T}}$ and the eigenvalue $\omega$ are associated with a prescribed value of $k$. This generalized eigenvalue problem is solved via an Arnoldi algorithm.

The adjoint eigenvector $\boldsymbol{q}^{+}$associated with the direct eigenvector $\boldsymbol{q}$ for a given value of $\omega$ is found as a solution of the discrete adjoint eigenvalue problem $\boldsymbol{A}^{\mathrm{H}} \boldsymbol{q}^{+}=\omega^{+} \boldsymbol{B}^{\mathrm{H}} \boldsymbol{q}^{+}$, with $\omega^{+}=\bar{\omega}$. The superscript $H$ denotes the conjugate transpose and an overbar denotes the complex conjugate. Note that $\boldsymbol{q}, \boldsymbol{q}^{+}$in the following always represent associated pairs with $\omega^{+}=\bar{\omega}$, and that $\boldsymbol{q}^{+}$is assumed to be normalized such that $\boldsymbol{q}^{+\mathrm{H}} \boldsymbol{B} \boldsymbol{q}=1$. The adjoint eigenvector can therefore be obtained by solving the linear system $\left(\boldsymbol{A}^{\mathrm{H}}-\bar{\omega} \boldsymbol{B}^{\mathrm{H}}\right) \boldsymbol{q}^{+}=0$, which is regularized with the normalization condition.

\subsection{Sensitivities}

Knowledge of $\boldsymbol{q}^{+}$allows one to explicitly compute how small variations of the matrix $\boldsymbol{A}$ translate to small variations in $\omega$. The perturbed direct problem

$$
(\boldsymbol{A}+\delta \boldsymbol{A})(\boldsymbol{q}+\delta \boldsymbol{q})=(\omega+\delta \omega) \boldsymbol{B}(\boldsymbol{q}+\delta \boldsymbol{q}),
$$

is linearized and multiplied with $\boldsymbol{q}^{+}$to give

$$
\boldsymbol{q}^{+\mathrm{H}}(\boldsymbol{A}-\omega \boldsymbol{B}) \delta \boldsymbol{q}+\boldsymbol{q}^{+\mathrm{H}}(\delta \boldsymbol{A}-\delta \omega \boldsymbol{B}) \boldsymbol{q}=0 .
$$

The first term in (2.5) is identically zero because $\boldsymbol{q}^{+\mathrm{H}}(\boldsymbol{A}-\omega \boldsymbol{B})=0$; the second term yields $\delta \omega=\boldsymbol{q}^{+\mathrm{H}} \delta \boldsymbol{A} \boldsymbol{q}$. In the context of this study, variations $\delta \boldsymbol{A}$ may arise from variations of the discrete baseflow vectors $\left(\boldsymbol{u}_{b}, \boldsymbol{\rho}_{b}\right)$ and of the wavenumber $k$. The associated gradients of the complex frequency are

$$
\frac{\partial \omega}{\partial \boldsymbol{u}_{b}}=\boldsymbol{q}^{+\mathrm{H}} \frac{\partial \boldsymbol{A}}{\partial \boldsymbol{u}_{b}} \boldsymbol{q}, \quad \frac{\partial \omega}{\partial \boldsymbol{\rho}_{b}}=\boldsymbol{q}^{+\mathrm{H}} \frac{\partial \boldsymbol{A}}{\partial \boldsymbol{\rho}_{b}} \boldsymbol{q}, \quad \frac{\partial \omega}{\partial k}=\boldsymbol{q}^{+\mathrm{H}} \frac{\partial \boldsymbol{A}}{\partial k} \boldsymbol{q} .
$$

The partial derivatives of $\boldsymbol{A}$ are obtained by differentiation of the left-hand sides of (2.1)-(2.3). The gradient vectors $\partial \omega / \partial \boldsymbol{u}_{b}$ and $\partial \omega / \partial \boldsymbol{\rho}_{b}$ in (2.6) reflect the sensitivity of $\omega$ to baseflow variations at the collocation points; their numerical values are inherently dependent on the grid spacing. For the purpose of $\S 4$, it is preferable to define sensitivity vectors $\boldsymbol{s}_{u}, \boldsymbol{s}_{\rho}$ as discrete representations of continuous, grid-independent functions $s_{u}(r), s_{\rho}(r)$ over the interval $0 \leqslant r \leqslant r_{\text {max }}$, such that

$$
\delta \omega=\int_{0}^{r_{\max }}\left[s_{u}(r) \delta u_{b}(r)+s_{\rho}(r) \delta \rho_{b}(r)\right] \mathrm{d} r \approx \boldsymbol{s}_{u}^{\mathrm{T}} \boldsymbol{M} \delta \boldsymbol{u}_{b}+\boldsymbol{s}_{\rho}^{\mathrm{T}} \boldsymbol{M} \delta \boldsymbol{\rho}_{b} .
$$


With a suitable diagonal metric $\boldsymbol{M}$, the grid-independent sensitivity values at the collocation points are then recovered as

$$
\boldsymbol{s}_{u}=\boldsymbol{M}^{-1} \frac{\partial \omega}{\partial \boldsymbol{u}_{b}}, \quad \boldsymbol{s}_{\rho}=\boldsymbol{M}^{-1} \frac{\partial \omega}{\partial \boldsymbol{\rho}_{b}} .
$$

The present formalism is entirely based on the discrete direct and adjoint dispersion relations. Continuous formulations of similar problems are given by Bottaro et al. (2003) and Hwang \& Choi (2006). Both approaches are expected to give identical results.

\subsection{Absolute eigenmode}

The scalar quantity $\partial \omega / \partial k=v_{g}$ is the group velocity of the direct eigenmode. The absolute instability mode is characterized by a zero group velocity. The possibility of obtaining $v_{g}$ directly from (2.6) leads to a new procedure for the computation of the absolute eigenmode for a given baseflow. Starting from an initial guess $k^{*}$ for the absolute wavenumber, the associated frequency and group velocity are computed by solving the direct and adjoint dispersion relations. A secant method is then employed to drive $v_{g}\left(k^{*}\right)$ towards zero, and to thereby converge to the true absolute wavenumber, $k_{0}$, and frequency, $\omega_{0}$. This procedure is found to be highly efficient in the present calculations. The secant method gives a fast rate of convergence, because the group velocity is a nearly linear function of $k$ in the vicinity of $k_{0}$. Each iteration involves the solution of one eigenvalue problem via an Arnoldi algorithm, which takes a fraction of a second even for excessive spatial resolutions, and the solution of a linear system for the adjoint eigenvector.

However, this algorithm does not guarantee that the final $\left(k_{0}, \omega_{0}\right)$ pair represents a pinch point between a $k^{+}$and a $k^{-}$branch, according to the Briggs-Bers criterion (Huerre \& Monkewitz 1990). The validity of this requirement has been verified for all final results presented in this paper via a detailed inspection of the spatial branches, as demonstrated in Lesshafft \& Huerre (2007).

\section{Absolute/convective transition for hyperbolic-tangent baseflow profiles}

In the search for optimal velocity and density profiles that favour the onset of absolute instability at small density differences, a parametric study of analytical profile shapes is attempted first. Following the approach of Srinivasan, Hallberg \& Strykowski (2010), the familiar hyperbolic-tangent profiles of Michalke (1971) are extended in such a way that mixing layer thickness parameters $b_{u}$ and $b_{\rho}$ may be prescribed independently for the baseflow velocity, $u_{b}$, and density, $\rho_{b}$. Furthermore, an offset $d$ between the radial positions of both mixing layers is introduced. In a first step, let the baseflow be given by

$$
\begin{aligned}
& \tilde{u}_{b}(r)=\frac{1}{2}\left(1+\tanh \left[\frac{1}{4 b_{u}}\left(\frac{1}{r}-r\right)\right]\right), \\
& \tilde{\rho}_{b}(r)=\left\{S+\frac{1-S}{2}\left(1+\tanh \left[\frac{1}{4 b_{\rho}}\left(\frac{1}{r-d}-r+d\right)\right]\right)\right\}^{-1} .
\end{aligned}
$$

With the implicit definition of the jet radius in the above formulation, the parameter $b_{u}$ precisely represents the momentum shear-layer thickness. However, since nonanalytical profile shapes will be considered in $\S 4$, an explicit definition of the jet radius as a reference length scale will be used consistently throughout this study. The 


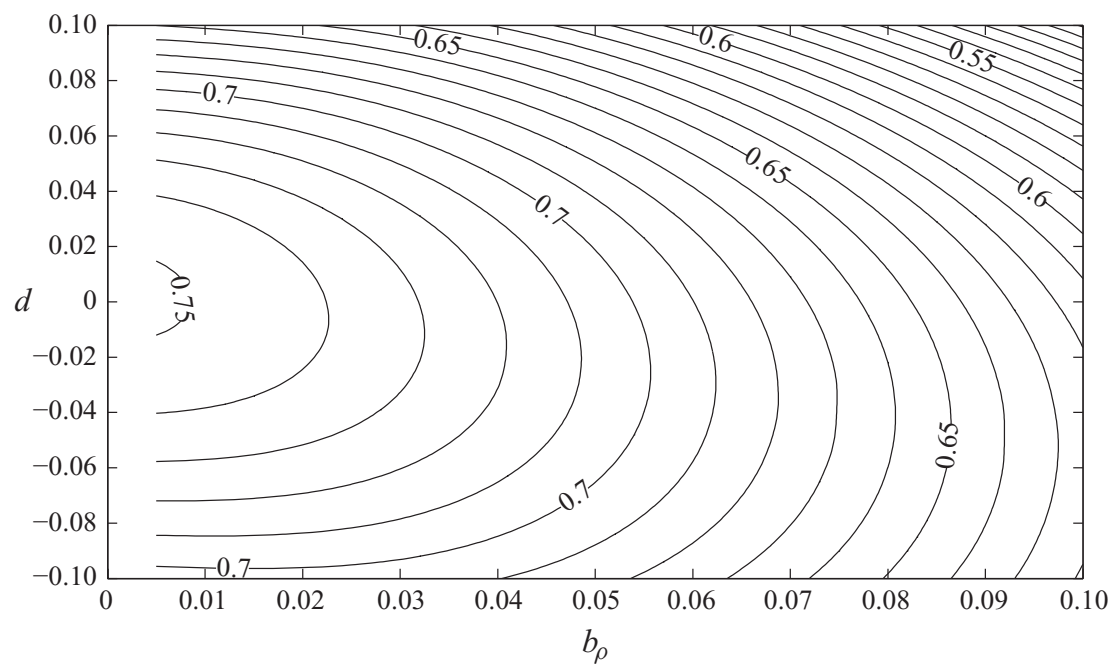

FIGURE 1. Critical density ratio $S_{\text {crit }}$ for absolute/convective transition of hyperbolic-tangent profiles (3.1) and (3.2) with $b_{u}=0.05$, as a function of $b_{\rho}$ and $d$.

length scale is adjusted in such a way that the non-dimensional volume flux of the baseflow is that of a plug profile with radius $1, \int_{0}^{\infty} u_{b}(r) r \mathrm{~d} r=0.5$. The profiles (3.1) and (3.2) are re-scaled as

$$
u_{b}(r)=\tilde{u}_{b}(\alpha r), \quad \rho_{b}(r)=\tilde{\rho}_{b}(\alpha r), \quad \text { with } \alpha^{2}=2 \int_{0}^{\infty} \tilde{u}_{b}(r) r \mathrm{~d} r .
$$

The momentum shear-layer thickness of the re-scaled baseflow is $\theta_{u}=b_{u} / \alpha$.

\subsection{Parametric mapping}

The effect of variations in $b_{\rho}$ and $d$ on the absolute/convective transition is systematically investigated for a fixed velocity profile parameter $b_{u}=0.05$ (corresponding to $R / \theta=20$ in Lesshafft \& Huerre 2007). Figure 1 displays contours of the critical density ratio $S_{\text {crit }}$, at which the transition from convective to absolute instability takes place, as a function of $b_{\rho}$ and $d$. The value of $S_{\text {crit }}$ for each parameter combination has been determined iteratively by use of a secant method, ensuring that $\omega_{0, i}\left(S_{\text {crit }}\right)=0$. Baseflows with a given combination $\left(b_{u}, b_{\rho}, d\right)$ are absolutely unstable for all $S<S_{\text {crit }}$ and convectively unstable for all $S>S_{\text {crit }}$.

Figure 1 clearly demonstrates that thin density mixing layers favour the onset of absolute instability. In the absence of radial offset, $d=0$, the critical density ratio increases monotonically with smaller values of $b_{\rho}$. The smallest value considered here is $b_{\rho}=0.005$, but it may be estimated by extrapolation that a discontinuous step profile for the temperature would yield an asymptotic critical ratio $S \approx 0.751$ for $b_{u}=0.05$ and $d=0$. The optimal radial offset at a given value of $b_{\rho}$ is found to be slightly negative. However, the increase in the critical density ratio that can be achieved by choosing $d \neq 0$ is rather insignificant, especially at small values of $b_{\rho}$. The general trends exposed in figure 1 for $b_{u}=0.05$ are also found to hold for values $b_{u}=0.1$ and 0.025 . 


\subsection{Parametric optimization}

In order to identify the optimal parameter combination $\left(b_{u}, b_{\rho}, d\right)$ that yields the highest possible value $S_{\text {crit }}$ for absolute/convective transition, one might proceed to extensively sample the four-dimensional parameter space, as done in the above section for one specific value of $b_{u}$. A more efficient approach may be derived from the sensitivity analysis of $\S 2$. The gradient of the complex frequency, $\omega$, with respect to $b_{u}, b_{\rho}, S$ and $d$ at any given point in the parameter space is easily constructed via the chain rule:

$$
\frac{\partial \omega}{\partial b_{u}}=\frac{\partial \omega}{\partial \boldsymbol{u}_{b}} \frac{\partial \boldsymbol{u}_{b}}{\partial b_{u}}, \quad \frac{\partial \omega}{\partial b_{\rho}}=\frac{\partial \omega}{\partial \boldsymbol{\rho}_{b}} \frac{\partial \boldsymbol{\rho}_{b}}{\partial b_{\rho}}, \quad \frac{\partial \omega}{\partial S}=\frac{\partial \omega}{\partial \boldsymbol{\rho}_{b}} \frac{\partial \boldsymbol{\rho}_{b}}{\partial S}, \quad \frac{\partial \omega}{\partial d}=\frac{\partial \omega}{\partial \boldsymbol{\rho}_{b}} \frac{\partial \boldsymbol{\rho}_{b}}{\partial d} .
$$

The gradients $\partial \omega / \partial \boldsymbol{u}_{b}$ and $\partial \omega / \partial \boldsymbol{\rho}_{b}$ are obtained from (2.6), the partial derivatives of $\boldsymbol{u}_{b}$ and $\boldsymbol{\rho}_{b}$ follow directly from (3.1) and (3.2).

The optimization algorithm starts with the computation of $\left(\omega_{0}, k_{0}\right)$ for a given parameter combination $\left(b_{u}, b_{\rho}, S, d\right)$. The gradient of the temporal growth rate $\omega_{i}=\operatorname{Im}(\omega)$ in the parameter space is evaluated according to the imaginary parts of (3.4). The parameters $b_{u}, b_{\rho}$ and $d$ are then varied in the direction of the positive gradient, such that $\omega_{i}$ is increased, whereas $S$ is varied in such a way that $\omega_{i}$ is driven towards zero. The absolute frequency/wavenumber pair is computed for the new baseflow, and the procedure is repeated until all four parameters are converged. The final value of $S$ represents the highest possible value at which absolute/convective transition can be achieved with a baseflow of the form (3.1) and (3.2).

As may be expected from the results presented in figure 1, the optimization drives the temperature mixing layer thickness to ever smaller values. In order to maintain an appropriate discrete resolution of the continuous temperature profile, a finite lower bound must be imposed for $b_{\rho}$. With two different restrictions $b_{\rho} \geqslant 0.005$ and $b_{\rho} \geqslant 0.0025$, the optimized critical temperature ratio is found to be

$$
S_{\text {crit }}=0.7514 \text { for } b_{u}=0.04935, \quad b_{\rho}=0.005, \quad d=0.0017
$$

and

$$
S_{\text {crit }}=0.7520 \quad \text { for } b_{u}=0.04929, \quad b_{\rho}=0.0025, \quad d=0.0021 .
$$

The value $b_{u}=0.05$, used for the parametric study presented in figure 1 , therefore is very near the optimal setting. The above optimization results are found to be insensitive to the choice of initial parameters, and in all likelihood represent the global optimum.

The threshold value $S_{\text {crit }}=0.752$, above which absolute instability is impossible for the present family of baseflow profiles, is to be compared to the value $S_{\text {crit }}=0.713$ that has been determined in previous studies of hyperbolic-tangent profiles restricted by the Crocco-Busemann law. The difference between these two values is not very significant, and it certainly does not explain the results of Coenen et al. (2008), who found absolute instability at $S=0.9$.

\section{Absolute/convective transition for shape-optimized profiles}

For a more general investigation of optimal conditions for the onset of absolute instability in jets, the limiting assumption of any parametrized analytical shape for the velocity and density profiles is now relaxed. Each discretization point in both profiles now represents one degree of freedom. A minimal set of constraints is imposed 

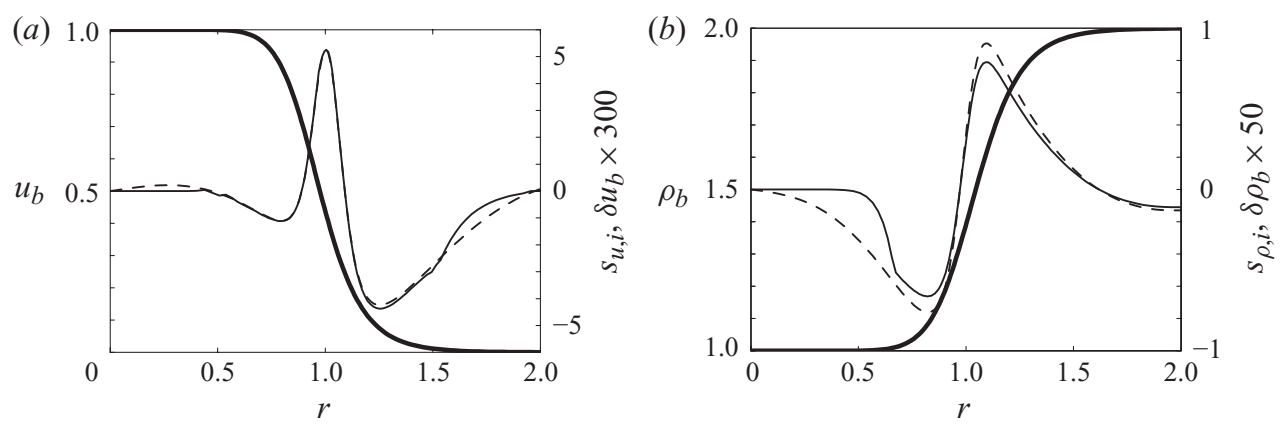

Figure 2. Baseflow profiles (3.1) and (3.2) with parameters $b_{u}=b_{\rho}=0.1, d=0, S=0.5$, constrained optimal variations and sensitivities of the absolute growth rate. $(a)-$, velocity $u_{b} ;$ - variation $\delta u_{b} ;--$, sensitivity $s_{u, i}=\operatorname{Im}\left(s_{u}\right) ;(b)$-, density $\rho_{b} ;$ - , variation $\delta \rho_{b} ;--$, sensitivity $s_{\rho, i}=\operatorname{Im}\left(s_{\rho}\right)$.

in order to ensure that the baseflow retains the basic characteristics of a jet with prescribed density ratio, $S$ :

(a) $0 \leqslant u_{b} \leqslant 1$ and $1 \leqslant \rho_{b} \leqslant S^{-1}$ for all $r$,

(b) only one inflection point in $u_{b}$ and in $\rho_{b}$,

(c) $\partial_{r} u_{b}=\partial_{r} \rho_{b}=0$ at $r=0$ and at $r_{\max }$.

Conditions $(b)$ and $(c)$ also imply that both profiles vary monotonically in $r$.

Within these limits, the profile shapes with the maximum absolute growth rate for a given value of $S$ are sought via successive baseflow modifications $\boldsymbol{u}_{b}^{n+1}=\boldsymbol{u}_{b}^{n}+\delta \boldsymbol{u}_{b}$ and $\boldsymbol{\rho}_{b}^{n+1}=\boldsymbol{\rho}_{b}^{n}+\delta \boldsymbol{\rho}_{b}$. This iterative process constitutes the outer optimization procedure. In each step, variations $\delta \boldsymbol{u}_{b}$ and $\delta \boldsymbol{\rho}_{b}$ of the current baseflow vectors $\boldsymbol{u}_{b}^{n}$ and $\boldsymbol{\rho}_{b}^{n}$ are obtained by solving an inner optimization problem: optimal variations are sought that maximize the increase of the temporal growth rate, given by the imaginary part of (2.7), while at the same time the modified profiles $\boldsymbol{u}_{b}^{n+1}$ and $\boldsymbol{\rho}_{b}^{n+1}$ must obey all the above constraints. This constrained optimization problem is solved using an sequential quadratic programming (SQP) method, as implemented in the Matlab routine fmincon. Bounds for $\delta \boldsymbol{u}_{b}$ and $\delta \boldsymbol{\rho}_{b}$ follow from $(a)$. The single-inflection-point condition is formally expressed as a linear inequality constraint, by requiring the second derivative of $\boldsymbol{u}_{b}^{n}+\delta \boldsymbol{u}_{b}$ to be non-positive for $r<r_{i p}-\varepsilon$ and non-negative for $r>r_{i p}+\varepsilon$, where $r_{i p}$ is the current position of the inflection point. This position is allowed to change by a distance $\varepsilon$ in the updated profile $\boldsymbol{u}_{b}^{n+1}$. A similar constraint is imposed on the density variations. Condition $(c)$ yields Neumann boundary conditions for $\delta \boldsymbol{u}_{b}$ and $\delta \boldsymbol{\rho}_{b}$. In addition, the norm of the variations is prescribed as a nonlinear constraint $\delta \boldsymbol{u}_{b}^{\mathrm{T}} \boldsymbol{M} \delta \boldsymbol{u}_{b}=\delta \boldsymbol{\rho}_{b}^{\mathrm{T}} \boldsymbol{M} \delta \boldsymbol{\rho}_{b}=0.01$ for well posedness.

Figure 2 illustrates the approach for a hyperbolic-tangent baseflow (3.1) and (3.2). The baseflow profiles (thick lines) are shown together with their sensitivity distributions (dashed lines) and optimal variations (thin solid lines, note the ordinate scaling). The sensitivity values represent the gradient of the temporal growth rate with respect to unconstrained baseflow changes. The optimal variations are seen to closely follow the gradient direction, except in regions where such baseflow modifications would violate the constraints.

Once the optimal variations have been applied to the baseflow, a filter procedure $\tilde{f}\left(r_{i}\right)=0.25 f\left(r_{i-1}\right)+0.5 f\left(r_{i}\right)+0.25 f\left(r_{i+1}\right)$ is performed on $\boldsymbol{u}_{b}^{n+1}$ and $\boldsymbol{\rho}_{b}^{n+1}$, in order to ensure that the profiles remain sufficiently smooth for an accurate computation of their radial derivatives. The absolute frequency and wavenumber of the new baseflow 

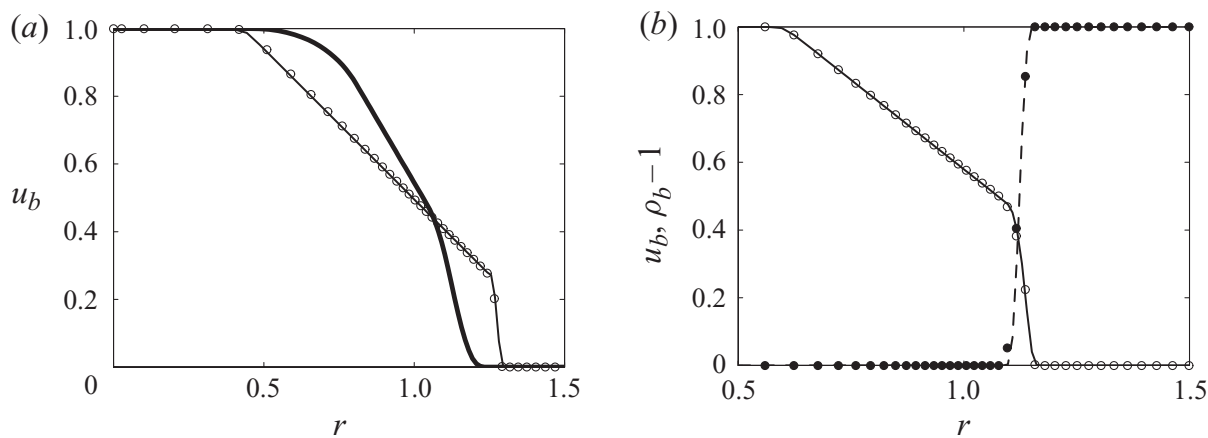

FIGURE 3. (a) Velocity profiles of a homogeneous jet at two stages of the optimization, starting from (3.1) with $b_{u}=0.1:-$, marginally unstable profile $\left(\omega_{0, i}=0\right) ;-$, optimal profile $\left(\omega_{0, i}=0.06\right)$. Circles: optimal profile reached from (3.1) with $b_{u}=0.05$. (b) Optimal baseflow profiles for a low-density jet with $S=0.5$, starting from $b_{u}=b_{\rho}=0.1$ : 一, velocity $u_{b}$; - density $\rho_{b}$. Circles: optimal profiles reached from (3.1) and (3.2) with $b_{u}=b_{\rho}=0.05$ (one out of two collocation points is plotted).

are then recalculated, and new variations are computed for the next outer optimization step.

\subsection{Optimized velocity profile of a homogeneous jet}

The case of a homogeneous jet is considered first. Keeping $\rho_{b} \equiv 1$ fixed, the baseflow velocity profile is optimized, starting from a hyperbolic-tangent profile (3.1) with $b_{u}=0.1$ and $\omega_{0}=1.21-0.19 \mathrm{i}, k_{0}=1.07-1.63 \mathrm{i}$. Successive modification of the velocity profile yields a steady increase of the absolute growth rate, which finally takes on positive values - i.e. the homogeneous jet becomes absolutely unstable.

The profile shape with zero absolute growth rate $\left(\omega_{0}=1.21, k_{0}=1.11-1.99 i\right)$ is presented in figure 3(a) as a thick line. Most notably, this profile is characterized by an asymmetry of the shear layer, which is partitioned into an inner region with low shear and an outer region with high shear. As the optimization is pursued further, this asymmetry becomes increasingly pronounced: the thin line in figure 3(a) represents the final result, with $\omega_{0, i}=0.06$. This optimal profile is of a nearly piece-wise linear shape, slightly smoothed by numerical filtering, with a sharp outer gradient. Without the single-inflection-point constraint, a concave shape would form in the inner region. The momentum shear-layer thickness changes during the optimization process from $\theta_{u}=0.10$ (initial profile with $\left.b_{u}=0.1\right)$ to values of $0.09\left(\omega_{0, i}=0\right)$ and $0.16\left(\omega_{0, i}=0.06\right)$.

The optimization procedure converges to virtually identical final results from different starting conditions. This is demonstrated by the circle symbols in figure $3(a)$, which represent the optimal result reached from an initial analytical profile with $b_{u}=0.05$ (one out of two collocation points is shown).

As neither density inhomogeneities nor counterflow are present in the current configuration, the onset of absolute instability remains unexplained in the light of the existing literature. An interpretation is attempted by examining the absolute eigenmode. Figure 4 displays the eigenfunctions of streamwise velocity $|U(r)|$ and pressure $|P(r)|$ for the marginally absolutely unstable profile as solid lines. These are compared with the corresponding eigenfunctions, shown as dashed lines, of the analytical profile (3.1) with $b_{u}=0.1$. The pressure eigenfunctions (figure $4 a$ ) are very similar in both cases; they peak on the jet axis and decay monotonically in the radial direction. This shape is a characteristic of the jet column mode, as opposed 

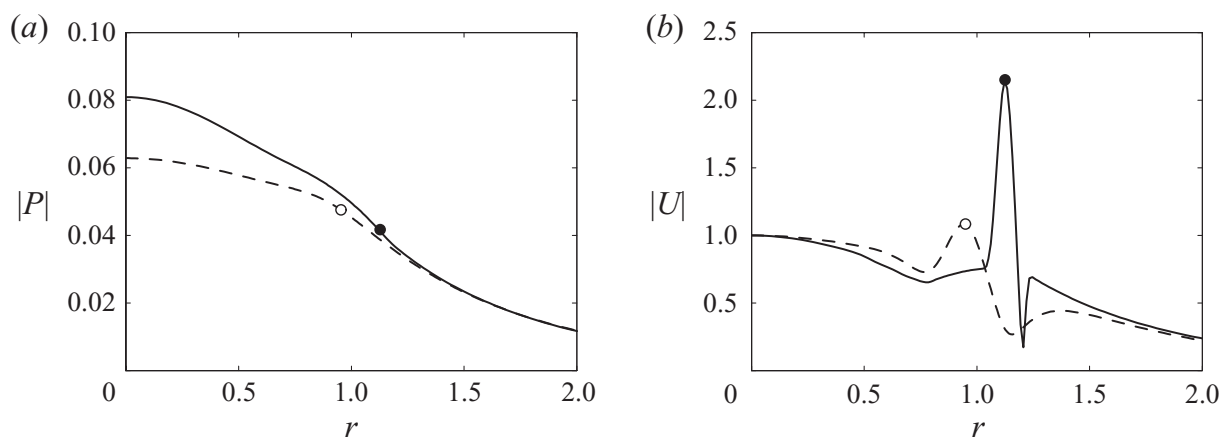

FIGURE 4. Eigenfunctions of the absolute mode in two homogeneous jets. - , marginally absolutely unstable profile (cf. figure $3 a$ ); - -, tanh-profile with $b_{u}=0.1$. ( $a$ ) Pressure $|P(r)|$; (b) streamwise velocity $|U(r)|$. Circles mark the respective locations of the maximum baseflow velocity gradient. All eigenfunctions are normalized with respect to the value of $|U|$ at $r=0$.

to the shear-layer mode in a round jet (Jendoubi \& Strykowski 1994). The velocity eigenfunctions (figure $4 b$ ) also share similar features; in particular, the location of the maximum amplitude in both cases coincides precisely with the maximum baseflow velocity gradient $(r=1.12$ and $r=0.95$, respectively). During the optimization procedure, this location continuously shifts into the region of low baseflow velocity. In analogy to configurations with counterflow, it may be surmised that a lower baseflow velocity around the peak location of the velocity eigenfunction leads to a lower group velocity of the instability mode, and thereby favours absolute instability.

\subsection{Optimized velocity and density profiles of a low-density jet}

When low-density jets are considered, the baseflow profiles of velocity and density may be varied separately or simultaneously. Starting from the analytical configuration displayed in figure 2, the baseflow density is first optimized alone, while the velocity profile is kept fixed. During the optimization, the baseflow density steadily evolves towards a step profile, with the maximum gradient always in the close vicinity of the maximum shear location. The absolute growth rate increases continuously with the steepening of the density profile. This observation is consistent with the results obtained for analytical profile shapes in $\S 3$.

Varying the baseflow velocity, while keeping the analytical density profile fixed leads to a piece-wise linear velocity profile similar to the one shown in figure $3(a)$. The presence of density variations increases the absolute growth rate, but it does not seem to alter in any significant way the trends discussed in $\S 4.1$.

Finally, $u_{b}$ and $\rho_{b}$ are allowed to evolve simultaneously, again starting from the analytical profiles given in figure 2. The final result is presented in figure $3(b)$. The velocity profile (solid line) again takes on a piece-wise linear shape, within the requirement of smooth discretization as enforced by the filter procedure, whereas the density distribution (dashed line) approaches a step profile. The maximum of the density gradient occurs at $r=1.12$, close to the location of maximum shear at $r=1.14$. These optimal profile shapes are again reached from various starting conditions: the circle symbols in figure $3(b)$ represent a nearly identical optimization result, obtained from an initial hyperbolic-tangent profile with $b_{u}=b_{\rho}=0.05, d=0$ and $S=0.5$. The absolute growth rate of the optimal baseflow is $\omega_{0, i}=0.225$, the shear-layer-momentum thickness is $\theta_{u}=0.095$. For comparison, the highest absolute growth rate that can be achieved with the analytical profile shapes (3.1) and (3.2) at 
$S=0.5$ is determined as $\omega_{0, i}=0.172$, for a much thinner shear layer $\theta_{u} \approx b_{u}=0.036$ and with $b_{\rho} \rightarrow 0, d=0$.

\section{Conclusions}

The onset of absolute instability in low-density jets has been investigated for a large variety of velocity- and density-profile shapes. The primary aim of this study was to determine whether a universal upper bound of the density ratio exists, above which absolute instability, in the absence of swirl or counterflow, would be impossible. The investigation is restricted to axisymmetric disturbances in inviscid, zero-Mach-number settings, and density ratios $S>1$ (heavy jets) are not considered.

First, a classical family of hyperbolic-tangent baseflow profiles was extended to allow for independently prescribed values of the velocity and density mixing-layer thickness, as well as for a radial offset between the two. The results of $\S 3$ have shown that only a modest increase of $S_{\text {crit }}$ from 0.713 to 0.752 can be achieved within the remaining restriction to hyperbolic-tangent profile shapes. In $\S 4$, velocity and density profiles were iteratively modified, according to the sensitivity of the absolute growth rate $\omega_{0, i}$, such as to maximize $\omega_{0, i}$ for a given density ratio. The most important result from this investigation is that certain velocity profiles may exhibit absolute instability even in the homogeneous case $S=1$. The precise shape of these profiles, as identified in $\S 4$, is certainly not encountered in typical laboratory jets. Yet their most characteristic feature, an asymmetry of the shear layer that shifts the maximum shear into the low-velocity region, may be expected to be present in common jet flows immediately downstream of the nozzle. In particular, the velocity profiles of Coenen et al. (2008), which largely motivated the present study, prominently display such asymmetric shear layers.

An interpretation of how shear-layer asymmetry may promote absolute instability is based on the observation that the peak of the velocity eigenfunction coincides with the location of maximum baseflow shear. It is argued that the group velocity of this mode is likely to decrease as its amplitude peak shifts into a region of low baseflow velocity.

Density profiles that optimally favour absolute instability have consistently been found to take the form of a step function. The step occurs very near the location of maximum shear.

All results presented herein lead to the conclusion that low-density jets may in principle display absolute instability at any density ratio. Furthermore, the momentum thickness of the velocity profile has been seen to be an inadequate measure for the instability characteristics. Whether a given baseflow is absolutely or convectively unstable depends critically on the precise shape of the velocity and density profiles.

We gratefully acknowledge the work of E. Obert, who carried out the calculations presented in figure 1 during her stay at LadHyX, with support from the MITFrance program. Valuable discussions with Y. Hwang and F. Martinelli have greatly contributed to this study. We thank P. Schmid and P. Huerre for their helpful comments on this manuscript.

\section{REFERENCES}

Bottaro, A., Corbett, P. \& Luchini, P. 2003 The effect of base flow variation on flow stability. J. Fluid Mech. 476, 293-302. 
Coenen, W., Sevilla, A. \& Sánchez, A. 2008 Absolute instability of light jets emerging from circular injector tubes. Phys. Fluids 20, 074104.

Gallaire, F. \& Chomaz, J.-M. 2003 Mode selection in swirling jet experiments: a linear stability analysis. J. Fluid Mech. 494, 223-253.

Hallberg, M., Srinivasan, V., Gorse, P. \& Strykowski, P. 2007 Suppression of global modes in low-density axisymmetric jets using coflow. Phys. Fluids 19, 014102.

Huerre, P. \& Monkewitz, P. 1990 Local and global instabilities in spatially developing flows. Annu. Rev. Fluid Mech. 22, 473-537.

Hwang, Y. \& CHOI, H. 2006 Control of absolute instability by basic-flow modification in a parallel wake at low Reynolds number. J. Fluid Mech. 560, 465-475.

Jendoubi, S. \& StRYKowski, P. 1994 Absolute and convective instability of axisymmetric jets with external flow. Phys. Fluids 6, 3000-3009.

Lesshafft, L. \& Huerre, P. 2007 Linear impulse response in hot round jets. Phys. Fluids 19, 024102.

Lesshafft, L., Huerre, P. \& Sagaut, P. 2007 Frequency selection in globally unstable round jets. Phys. Fluids 19, 054108.

Michalke, A. 1971 Instabilität eines kompressiblen runden Freistrahls unter Berücksichtigung des Einflusses der Strahlgrenzschichtdicke. Z. Flugwiss. 19, 319-328.

Monkewitz, P., Bechert, D., Barsikow, B. \& Lehmann, B. 1990 Self-excited oscillations and mixing in a heated round jet. J. Fluid Mech. 213, 611-639.

Monkewitz, P. \& Sohn, K. 1988 Absolute instability in hot jets. AIAA J. 26, 911-916.

Srinivasan, V., Hallberg, M. P. \& Strykowski, P. J. 2010 Viscous linear stability of axisymmetric low-density jets: parameters influencing absolute instability. Phys. Fluids 22 (2), 024103. 